\title{
A 3-year survey of acute poisoning exposures in infants reported in telephone calls made to the Tygerberg Poison Information Centre, South Africa
}

\author{
C J Marks, ${ }^{1}$ BSc Pharm, Hons Pharmacology, MSc MedSci; D J van Hoving, ${ }^{2}$ MB ChB, DipPEC, MMed (Em Med), \\ MSc MedSci (Clin Epi) \\ ${ }^{1}$ Division of Clinical Pharmacology, Department of Medicine, Faculty of Medicine and Health Sciences, Stellenbosch University, Cape Town,
South Africa \\ ${ }^{2}$ Division of Emergency Medicine, Stellenbosch University, Cape Town, South Africa
}

Corresponding author: C Marks (carinem@sun.ac.za)

\begin{abstract}
Background. Infants undergo rapid development changes and are particularly vulnerable to toxic chemicals. Identifying and evaluating the toxic risks that exist in this age group could be very valuable when making recommendations on how to prevent specific types of poisoning. Objectives. This study analysed the toxic substances responsible for acute poisoning exposures in infants $(<1$ year of age) as well as the severity of the exposures.

Methods. A retrospective analysis of the Tygerberg Poison Information Centre (TPIC) database was conducted over a 3-year period (1 January 2011 to 31 December 2013). Descriptive statistics are provided for the entire study population as well as for the neonatal subgroup ( $<30$ days old). Results. The TPIC handled 17434 consultations during the 3-year study period. Infants were involved in 1101 cases (6.3\%), of which 46 cases $(4.2 \%)$ were neonates. Most enquiries about infants were associated with non-drug chemicals $(n=824,74.8 \%)$. Pharmaceuticals were involved in 185 cases (16.8\%) followed by biological exposures (e.g. snake and spider bites, scorpion stings, plant and mushroom poisonings) ( $n=109,9.9 \%)$. Most infants $(n=987,89.6 \%)$ presented with no or only minor symptoms. In neonates, $17(37.0 \%)$ presented with moderate to severe toxicity. Six of these $(35.3 \%)$ were poisoned by complementary and alternative medicines.

Conclusion. Most poisoning exposures in infants are not serious and can be safely managed at home after contacting a poison centre. Identification and documentation of poisoning in this special population is of great importance.
\end{abstract}

S Afr J Child Health 2016;10(1):43-46. DOI:10.7196/SAJCH.2016.v10i1.1045

The diagnosis and management of poisoning in infants are challenging. Although fatalities among these young children are rare, poisoning still represents a frequent cause for hospital admission. ${ }^{[1]}$ One would expect that most poisonings in infants would be administered by a carer, but children are inquisitive by nature, and as infants become mobile ( 8 - 12 months), they start to explore their surroundings, inevitably putting things into their mouths. ${ }^{[2]}$ This causes accidental self-poisoning; however, the true extent of acute poisoning exposures in infants $(<1$ year of age) is not well documented. Although poisoning trends change with increasing age, the agents responsible for these poisonings are usually found in and around the home. ${ }^{[2,3]}$ Young children remain particularly vulnerable to poisoning, since exposure to a poison at a critical stage in their development may have severe long-term consequences for health.

Extrapolating from a population-based study in a developed country, ${ }^{[4]}$ we can estimate that about 500000 people might be poisoned each year in South Africa (SA). In 2004 the World Health Organization (WHO) estimated that unintentional poisoning caused 346000 deaths worldwide, of which $91 \%$ occurred in low- and middle-income countries. ${ }^{[5]}$ In 2012, unintentional injury, which included poisoning, was the fifth leading cause of death in infants in the USA. ${ }^{[6]}$ Unintentional injuries in young children are largely preventable. Infants cannot remove themselves from danger and cannot read warning signs and labels. This important information should lead to the development of preventive strategies.

Parents often seek medical attention when a child has been exposed to a poison, more so if the poisoning involves an infant or neonate. This special population is more susceptible to poisoning because of their pharmacokinetic differences, their small blood volume and small size. ${ }^{[7]}$ Identifying and evaluating the toxic risks that exist in this age group could be very valuable when making recommendations on how to prevent specific types of poisoning. We aimed to describe which toxic substances are responsible for acute poisoning exposures and the severity of the poisoning as reported to the Tygerberg Poison Information Centre (TPIC).

\section{Methods}

Study design

A retrospective analysis of the TPIC database was conducted over a 3-year period (1 January 2011 to 31 December 2013). This study was approved by the Health Research Ethics Committee of the University of Stellenbosch (Ref: S13/04/088).

\section{Study setting and data collection}

The TPIC is located in the Western Cape Province of SA and forms an integral part of the Tygerberg Academic Hospital. It is located in the Division of Clinical Pharmacology, Faculty of Medicine and Health Sciences, on the Tygerberg campus of Stellenbosch University. The TPIC provides a free toxicology service to healthcare workers and members of the public throughout SA. The service covers toxicity assessments, as well as poisoning treatment recommendations, and is available 24 hours a day. Poisoning consultations are handled by telephone on a dedicated emergency number, which is advertised nationally. Poisoning enquiries are answered by pharmacists, medical scientists or medical doctors. Calls received are manually recorded on a standard TPIC consultation form and include: date and time; geographical area; medical background of the caller; patient's age and gender; time, intent and route of exposure; substances involved; 
advice given; and the poisoning severity score. Severity grading is done according to the European Association of Poisons Centres and Clinical Toxicologists poisoning severity score (severity $0-1=$ no or minor symptoms; severity $2-3=$ moderate to severe toxicity) and is allocated to the patient at the time of the call, when the observed clinical symptoms and signs are taken into account. ${ }^{[8]}$ Data from consultation forms are entered on an electronic database by a permanent TPIC staff member; this is not cross-checked because of financial and time restraints.

\section{Study population}

All data in the TPIC database relating to infants ( $<1$ year old) were included for the period 1 January 2011 to 31 December 2013. In the event of incomplete consultation forms, the cases were not excluded; instead, only the specific missing variable was indicated as unknown.

\section{Data collection and analysis}

Variables collected from the TPIC database included: time of call; geographical area; caller's medical background; patient's age and gender; time, intent and route of exposure; substances involved; and the poisoning severity score. Collected data were committed to a standard Microsoft Excel (USA) spreadsheet. Descriptive statistics are provided for the entire study population as well for the neonatal subgroup ( $<30$ days old).

\section{Results}

The TPIC handled 17434 consultations during the 3-year study period. Infants were involved in 1101 cases (6.3\%), of which 46 cases $(4.2 \%)$ were neonates (Fig. 1). The mean age of the study population was 9.8 months.

Most poisoning enquiries were received from the Western Cape ( $n=384,34.9 \%$ ) (Fig. 2) and the majority were made by healthcare professionals $(n=659,59.9 \%)$ (Fig. 3 ).

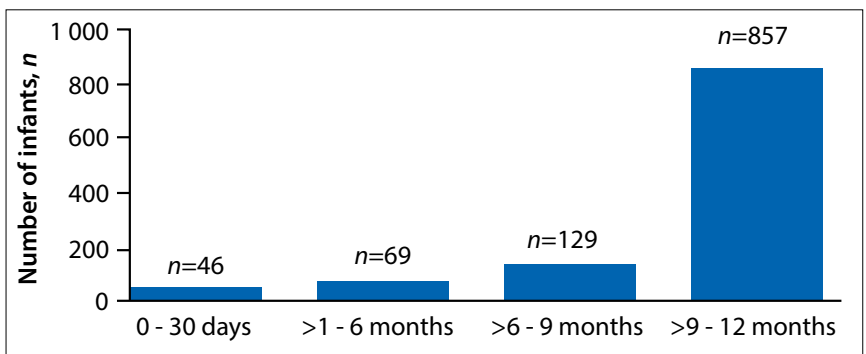

Fig. 1. Age distribution of infants exposed to poisons as reported to the TPIC $(\mathrm{N}=1$ 101).

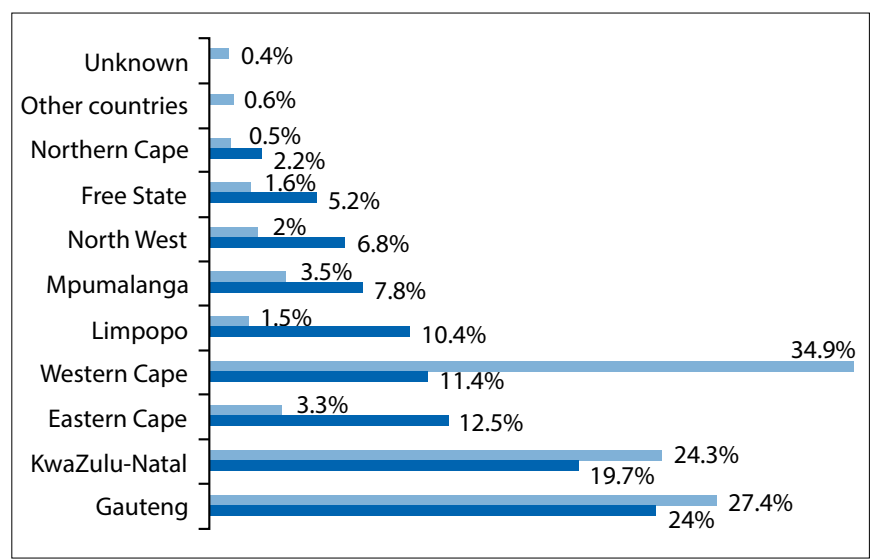

Fig. 2. Geographical distribution of calls received by the TPIC (light blue) v. SA's population per province (dark blue). ${ }^{\left[{ }^{[]}\right.}$
Almost all exposures were accidental ( $n=1092,99.2 \%)$ with only 8 cases $(0.7 \%)$ reported as deliberate. The gender was recorded in 1081 cases (males $n=591,54.7 \%$ ).

Most enquiries about infants were associated with non-drug chemicals $(n=824,74.8 \%)$. One hundred and sixty-eight $(15.4 \%)$ infants were exposed to pharmaceuticals, but often combinations of drugs were involved (e.g. antihistamine plus an analgesic). Pharmaceuticals were involved in 185 cases $(16.8 \%)$ followed by biological exposures (e.g. snake and spider bites, scorpion stings, plant and mushroom poisonings) ( $n=109,9.9 \%)$.

Irritants and corrosives were responsible for most non-drug chemical exposures $(n=255,30.9 \%)$ (Fig. 4). Toilet cleaner discs were liable for 45 exposures (17.6\%). One hundred and eightyfour exposures (22.3\%) were related to pesticides, of which almost a third related to rodenticides $(n=56,30.4 \%)$ and a quarter to pyrethroids $(n=45,24.5 \%)$. Of the rodenticides, $40(71.4 \%)$ involved anticoagulant rodenticides and $16(28.6 \%)$ to aldicarb, a cholinesterase inhibitor that is often illegally sold as a rat poison. ${ }^{[10]}$ Alcohols were responsible for 51 non-drug exposures (6.2\%); 31 involved surgical spirits (60.8\%). One hundred and two non-drug chemicals (12.4\%) were categorised as mildly toxic substances; 52 of these cases (55.3\%) involved silica gel. Of 109 biological exposures, plants and mushrooms were responsible for 80 cases $(73.4 \%)$.

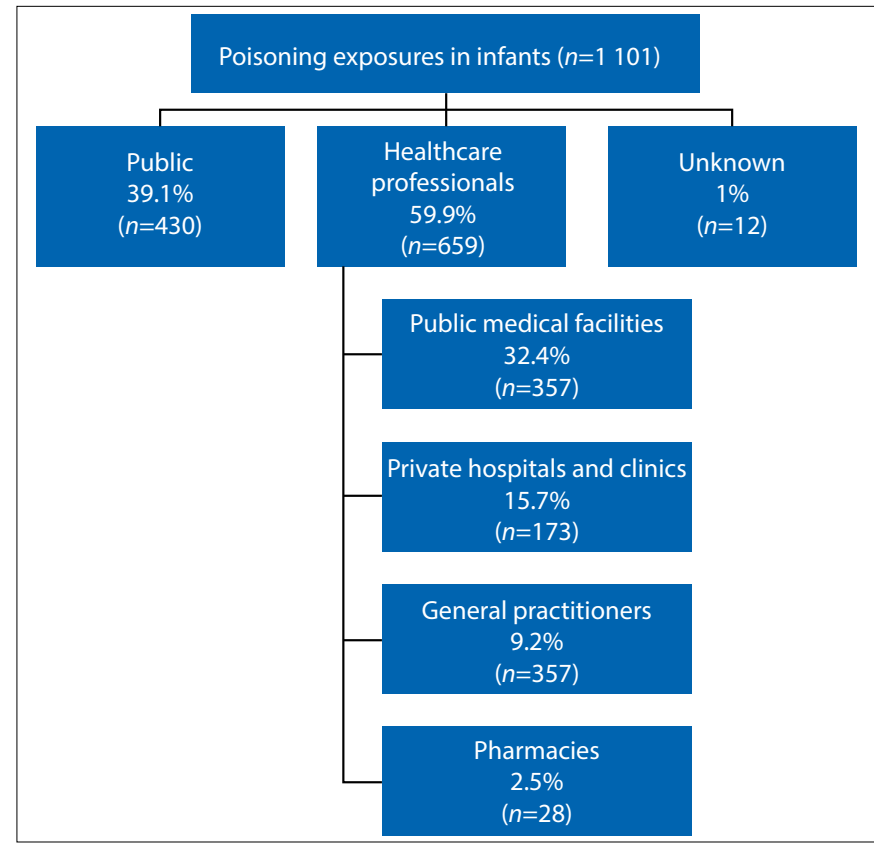

Fig. 3. Flow chart depicting the origin of calls received by the TPIC.

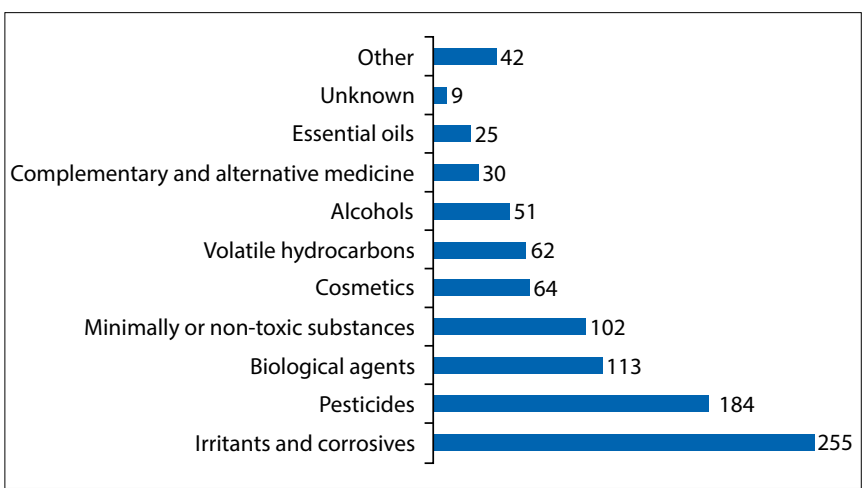

Fig. 4. Number of enquiries about chemical and biological non-drug exposures $(\mathrm{n}=933)$ in infants. 


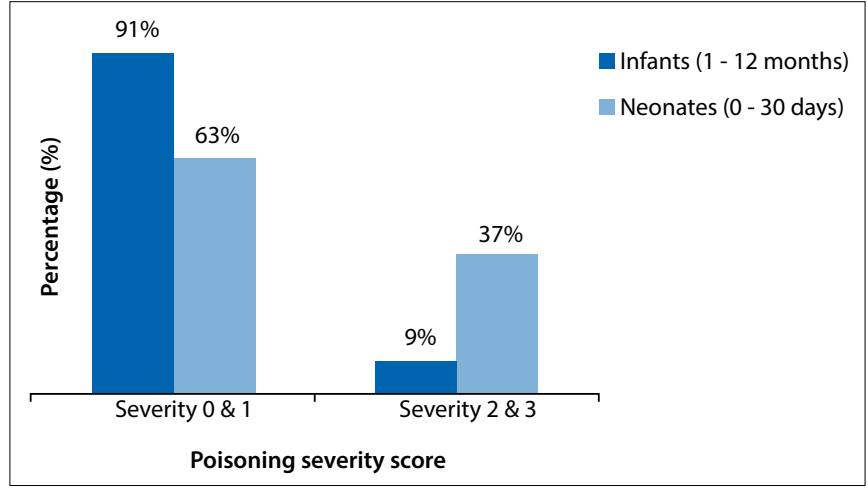

Fig. 5. Comparison of poisoning severity scores between neonates and older infants.

Table 1 shows the pharmaceuticals responsible for poisoning in infants, with analgesics being the most common group of drugs $(n=49,26.5 \%)$. Paracetamol was involved in 23 cases $(47.0 \%)$.

Most infants $(n=987,89.6 \%)$ presented with no or only minor symptoms. No initial deaths were recorded; however, fatality cannot be excluded since poisoned patients were not followed up. In neonates, $17(37.0 \%)$ presented with moderate to severe toxicity. Six of these $(35.3 \%)$ were poisoned by complementary and alternative medicines (CAM) (Table 2); four received SA-sold 'Dutch remedies' that contained herbal products and two received SA traditional medicines that contained unknown herbal preparations and plant extracts. Fig. 5 depicts the differences in the poisoning severity score between neonates and older infants.

\section{Discussion}

Between 1 January 2011 and 31 December 2013 the TPIC received 17434 poisoning-related calls. Of these, 1101 (6.3\%) involved infants. Our findings are similar to USA data where exposures involving infants ranged between $5.3 \%$ and $10.5 \% .{ }^{[1,12]}$ These USA data are derived from the American Association of Poison Control Centres which received and managed poisoning enquiries similar to those handled by the TPIC.

SA is divided into nine provinces, but most calls came from only three ( $n=954 ; 86.6 \%)$ (Fig. 2). The skewed presentation of calls from the Western Cape, KwaZulu-Natal and Gauteng could be due to these provinces having larger populations, but it does not explain why we received only a few enquiries from the Eastern Cape. One reason may be that people can only call a poison information centre if they know that one exists! ${ }^{[13]}$ The Western Cape has two poison centres. Both KwaZulu-Natal and Gauteng once had poison centres which have now closed. In addition, most of the calls were received from healthcare professionals (60\%). Educational standards in SA are low and may make it likely that parents would be unaware of a poisons information centre, so would take children directly to a healthcare facility. The opposite happens in the USA, where poison information services are well known and integrated into emergency services. ${ }^{[1]}$

Childhood poisoning exposures are almost always accidental. ${ }^{[14]}$ Deliberate poisoning in children is unlikely to be reported to poison centres. A more accurate estimate of the prevalence of intentional poisoning in infants would be obtained from hospital admissions, as well as those from primary healthcare facilities. Eight children were poisoned intentionally during the study period. Two cases included intrauterine exposure to a chemical. It is not known when exactly in the gestation period these prenatal exposures took place, but both babies were born soon after the mother was exposed to the toxin. In six cases, poisoning was deliberate. The detection of intentional poisoning is worrying as this is a rare phenomenon. ${ }^{[15]}$ Socioeconomic factors might play a role and require further investigation.
Table 1. Pharmaceuticals responsible for poisoning exposures in infants $(N=185)$

\begin{tabular}{ll}
\hline Drug categories & $n(\%)$ \\
\hline Analgesics & $49(26.5)$ \\
Antihistamines & $18(9.7)$ \\
Antimicrobials & $17(9.2)$ \\
Neuroleptics (antipsychotic drugs) & $11(5.9)$ \\
Antidepressants & $9(4.9)$ \\
Hypnotics & $8(4.3)$ \\
Antihypertensives & $7(3.8)$ \\
Anti-epileptics & $7(3.8)$ \\
Vitamins and minerals & $6(3.2)$ \\
Miscellaneous & $53(28.7)$
\end{tabular}

Table 2. Infants exposed to complementary and alternative medicine $(N=30)$

\begin{tabular}{lll}
\hline & Severity 0 - 1, ${ }^{*} \boldsymbol{n}(\%)$ & Severity 2 - 3, $\boldsymbol{n}(\%)$ \\
\hline $\begin{array}{l}\text { Neonates, }<1 \text { month } \\
\text { of age }(n=10)\end{array}$ & $4(40)$ & $6(60)$ \\
$\begin{array}{l}\text { Infants, } 1-12 \\
\text { months of age }(n=20)\end{array}$ & $14(70)$ & $6(30)$ \\
& * Severity $0-1$ = no or minor symptoms; severity 2 - 3 = moderate to severe toxicity. ${ }^{[8]}$
\end{tabular}

Our data revealed that non-drug exposures were responsible for almost $85 \%$ of reported cases. The three most commonly implicated non-drug toxins were irritant and corrosive substances, followed by pesticides and biological exposures. The high prevalence of toilet cleaner discs exposure ( $n=45,17.6 \%$ of exposures to irritants and corrosives) was an interesting finding. Although most toilet detergents, household soaps and bleaches have irritant properties, it is impossible to predict whether these agents will cause injury to the oesophagus and stomach. There is also a frustrating lack of information in respect of potentially toxic ingredients contained in commercially available household preparations. Labels on such products seldom provide adequate information on ingredients, and often do not contain warnings about their potential toxicity. Owing to the significant public ignorance regarding the toxicity of household non-drug chemicals, these preparations should not be displayed close to foodstuffs or within reach of children (as is often the case in households and supermarkets). ${ }^{[16]}$

Pesticide exposures are a major public health problem in SA, ${ }^{[10]}$ predominantly in settings of low education and poor regulatory frameworks. A previous study investigated accidental pesticide poisoning in children. ${ }^{[10]}$ Poor and crowded areas are a breeding ground for most pests, and people seek cheap effective ways to deal with the problem. They often buy rodenticides intended for agricultural use instead of home use from street vendors (agricultural pesticides have a higher toxicity). In our study 16 infants were exposed to aldicarb, an illegally sold rodenticide.

Compared with exposures to biological toxic substances produced by animals $(n=29)$, enquiries regarding poisoning exposures to plants and mushrooms were relatively high in our study $(n=80)$. The inherent toxicity of most plants is low, so the ingestion of small to moderate quantities is unlikely to produce toxic effects. Serious poisonings caused by plants are exceptional as a young child eats only a few leaves or seeds at a time.

In recent years there has been an increase in the popularity of CAM. This type of medicine includes medical products (e.g. herbal medicine, 
homeopathy, traditional medicine) and medical practices (e.g. body manipulation, acupuncture) that are not part of standard medical care. Unlike conventional medicine, some CAM has not been through clinical testing and is often not safe. ${ }^{[17]}$ It is regularly given to newborns to treat a diversity of symptoms, including colic. Owing to their small size and differences in pharmacokinetics, neonates are at an increased risk for toxicity. ${ }^{[7]}$ In our study six neonates were seriously poisoned by CAM (Table 2). Two involved SA traditional medicine (a combination of animal, mineral and plant products to induce physiological or psychological healing effects) and four involved herbal products (a combination of plant seeds, berries, roots, leaves, bark, or flowers for medicinal purposes). Three of the four herbal products contained valerian which has been associated with toxicity ${ }^{[18]}$ In this study serious CAM-related poisonings occurred more in neonates (60\%) compared with older infants (30\%) (Table 2). The low weight and physiological differences in this vulnerable population could be a contributing factor. ${ }^{[7]}$

\section{Study strengths}

This study is valuable because data on poisoning are hard to obtain anywhere. They are particularly sparse in developing countries, such as SA. On the African continent there are very few poison information centres from which data on telephone calls can be obtained.

\section{Study limitations}

Incomplete data might have influenced the results in either direction. However, the possible effect was limited by only indicating the specific missing variable as unknown and not excluding the entire case.

Our study only includes data from one poison centre in SA, and cannot be extrapolated to estimate the prevalence of poisoning in SA. Data on admissions to hospitals for acute poisoning are very different from data recorded from telephone enquiries by poison information centres. Telephone enquiries reflect a need for information for professionals and for the public. Future epidemiological studies of poisoning should use data from telephone enquiries and from admissions to hospital.

No cases reported to the TPIC were officially followed up because of time and financial constraints. Data regarding measurable clinical outcomes (e.g. morbidity and mortality) are therefore lacking and the clinical significance of the data remains unclear.

\section{Conclusion}

Most poisoning exposures in infants are not serious and can be safely managed at home after contacting a poison centre. Poisoning is more challenging in neonates because of physiological, behavioural and biological differences. Identification and documentation of poisoning in this special population is of great importance. The use of CAM in infants can be harmful and more information is needed to ensure its safe use.
Acknowledgements. We would like to express our sincere thanks and appreciation to the Tygerberg Poison Information Centre staff.

\section{References}

1. Azkunaga B, Mintegi S, Bizkarra I, Ferna'ndez J. Toxicology surveillance system of the Spanish Society of Paediatric Emergencies: First-year analysis. Eur J Emerg Med 2011;18(5):285-287. [http://dx.doi.org/10.1097/ MEJ.0b013e3283462504]

2. Chatsantiprapa K, Chokkanapitak JPN. Host and environment factors for exposure to poisons: A case-control study for preschool children. Thailand Inj Prev 2001;7:214-217.

3. Agran PF, Anderson C, Winn D, Trent R, Walton-Haynes L, Thayer S. Rates of pediatric injuries by 3 -month intervals for children 0 to 3 years of age. Pediatrics. 2003;111(6):e683-e692. http://pediatrics.aappublications.org/ content/111/6/e683.long (accessed 15 January 2015).

4. Kristinsson J, Palsson R, Gudjonsdottir GA, Blondal M, Gudmundsson S, Snook CP. Acute poisonings in Iceland: A prospective nationwide study. Clin Toxicol (Phila) 2006;46:126-132.

5. World Health Organization. Global Health Observatory Data Repository. Geneva: World Health Organization, 2009. http://apps.who.int/gho/data/?theme=main (accessed 16 August 2015).

6. Centers for Disease Control and Prevention, National Center for Injury Prevention and Control. Injury Prevention and Control: Data and Statistics. Ten Leading Causes of Death and Injury in United States, 2015. http://www. cdc.gov/injury/wisqars/leadingcauses.html (accessed 23 February 2015).

7. Ginsberg G, Hattis D, Sonawane B. Incorporating pharmacokinetic differences between children and adults in assessing children's risks to environmental toxicants. Toxicol Appl Pharmacol 2004;198(2):164-183. http://www.ncbi.nlm. nih.gov/pubmed/15236952 (accessed 18 January 2015).

8. Persson HE, Sjöberg GK, Haines JA, Pronczuk de Garbino J. Poisoning Severity Score grading of acute poisoning. J Toxicol Clin Toxicol 1998;36(3):205-213.

9. South Africa: Mid-year population estimates 2014. http://beta2.statssa.gov.za/ publications/P0302/P03022014.pdf (accessed 23 February 2015).

10. Balme KH, Roberts JC, Glasstone $\mathrm{M}$, et al. Pesticide poisonings at a tertiary children's hospital in South Africa: An increasing problem. Clin Toxicol (Phila) 2010;48:928-934. [http//:dx.doi.org/10.3109/15563650.2010.534482]

11. American Association of Poison Control Centers. National Poison Data System. Annual Report 2013. http://www.aapcc.org/data-system (accessed 22 January 2015)

12. Franklin RL, Rodgers GB. Unintentional child poisonings treated in United States hospital emergency departments: National estimates of incident cases, population-based poisoning rates, and product involvement. Pediatrics 2008;122:1244-1251. [http//:dx.doi.org/10.1542/peds.2007-355]

13. Litovitz T, Benson BE, Youniss J, Metz E. Determinants of U.S. poison center utilization. Clin Toxicol (Phila) 2010;48(5):449-457. [http//:dx.doi. org/10.3109/15563651003757947]

14. Even KM, Armsby CC, Bateman ST. Poisonings requiring admission to the pediatric intensive care unit: A 5-year review. Clin Toxicol (Phila) 2014;52(5):519-524. [http//:dx.doi.org/10.3109/15563650.2014.909601]

15. Dine MS, McGovern ME. Intentional poisoning of children - an overlooked category of child abuse: Report of seven cases and review of the literature. Pediatrics 1982;70(1):32-35.

16. Savage EP, Tessari JD, Laurier P. Pesticides sold in grocery stores. Health Services Reports 1972;87(8):734-736

17. Niggemann B, Grüber C. Side-effects of complementary and alternative medicine. Allergy 2003;58(8):707-716. [http//:dx.doi.org/10.1034/j.13989995.2003.00219.x]

18. Tomassoni AJ, Simone K. Herbal medicines for children: An illusion of safety? Curr Opin Pediatr 2001;13(2):162-169. [http//:dx.doi.org/10.1097/00008480200104000-00014] 